\title{
ON THE BLOW-UP BEHAVIOR OF SOLUTIONS TO A SYSTEM WITH FRACTIONAL POWER
}

\author{
Ali Hakem \\ Departement D'informatique, Faculte de Technologie, 22000 Sidi Bel Abbes, Algerie
}

Received 2010-08-22, Revised 2012-11-08; Accepted 2013-06-28

\begin{abstract}
The aim of this research is to study the nonexistence of global weak solutions for systems of fractional power. The method relies on a suitable choice of test function.
\end{abstract}

Keywords: Nonexistence Global, Critical Exponent

\section{INTRODUCTION}

Since the articles of Fujita (1966) and OSEU (1968), critical exponents have attracted the attention of a sizable number of researchers. Fore valuable surveys of Fujita type theorems for equations as well as for systems of reaction-diffusion equations we refer to Levine (1990); Samarskii et al. (1995); Bandle and Brunner (1998) and Levine (1990). Escobedo and Herrero (1991) considered the system:

$$
\text { (S) }\left\{\begin{array}{l}
\mathrm{u}_{\mathrm{t}}=\delta \Delta \mathrm{u}+\mathrm{u}^{\mathrm{p}}, \quad \mathrm{v}_{\mathrm{t}}=\Delta \mathrm{v}+\mathrm{v}^{\mathrm{q}}, \quad \mathrm{t}>0, \mathrm{x} \in \mathrm{IR}^{\mathrm{n}} \\
\mathrm{u}(0, \mathrm{x})=\mathrm{u}_{0}(\mathrm{x}) \geq 0, \quad \mathrm{v}(0, \mathrm{x})=\mathrm{v}_{0}(\mathrm{x}) \geq 0 \\
\mathrm{u}_{0}, \mathrm{v}_{0} \in \mathrm{L}^{\infty}\left(\mathrm{IR}^{\mathrm{n}}\right)
\end{array}\right.
$$

with $\delta=1, \mathrm{p}, \mathrm{q}>0$, they derived global existence and blow-up results for $(\mathrm{S})$. They showed that all positive solutions of (S) blow-up in finite time for:

$$
\mathrm{pq}>1 \text { and } \frac{\mathrm{n}}{2} \leq \frac{\max (\mathrm{p}, \mathrm{q})+1}{\mathrm{pq}-1}
$$

Then in Fila et al. (1994) extended the results to the case where $0 \leq \delta \leq 1$. They use the same technique as in (Escobedo and Herrero, 1991) and a property satisfied by the heat kernel. In a recent paper, Igbida and Kirane (2002) considered, with respect to the nonexistence of global solutions, the more general system:

$$
(\mathrm{IK})\left\{\begin{array}{l}
\mathrm{u}_{\mathrm{t}}+(-\Delta)\left(\mathrm{a}_{11} \mathrm{u}\right)=\mathrm{h}(\mathrm{t}, \mathrm{x})|\mathrm{v}|^{\mathrm{p}} \\
\mathrm{v}_{\mathrm{t}}+(-\Delta)\left(-\mathrm{a}_{21} \mathrm{u}\right)+(-\Delta)\left(\mathrm{a}_{22} \mathrm{v}\right)=\mathrm{k}(\mathrm{t}, \mathrm{x})|\mathrm{w}|^{\mathrm{q}} \\
\mathrm{w}_{\mathrm{t}}+(-\Delta)\left(\mathrm{a}_{31} \mathrm{u}\right)+(-\Delta)\left(\mathrm{a}_{32} \mathrm{v}\right) \\
+(-\Delta)\left(\mathrm{a}_{33} \mathrm{w}\right)=1(\mathrm{t}, \mathrm{x})|\mathrm{u}|^{\mathrm{r}} \\
\mathrm{u}(0, \mathrm{x}) \geq 0, \quad \mathrm{v}(0, \mathrm{x}) \geq 0, \quad \mathrm{w}(0, \mathrm{x}) \geq 0
\end{array}\right.
$$

for $\mathrm{p}, \mathrm{q}, \mathrm{r}>0, \mathrm{a}_{\mathrm{ij}}$ measurable, positive and bounded functions. The conditions on $\mathrm{h}, \mathrm{k}$ and 1 required are:

$$
\left\{\begin{array}{l}
0<\mathrm{h}\left(\mathrm{R}^{2} \tau, \mathrm{Ry}\right)=\mathrm{O}\left(\mathrm{R}^{\mu}\right) \\
0<\mathrm{k}\left(\mathrm{R}^{2} \tau, \mathrm{Ry}\right)=\mathrm{O}\left(\mathrm{R}^{\mathrm{k}}\right) \\
0<\mathrm{l}\left(\mathrm{R}^{2} \tau, \mathrm{Ry}\right)=\mathrm{O}\left(\mathrm{R}^{\lambda}\right)
\end{array}\right.
$$

Let us note in passing that Renclawowicz studied the completely coupled Fujita-type system:

$$
(\mathrm{R})\left\{\begin{array}{cl}
\mathrm{u}_{\mathrm{t}}=\Delta \mathrm{u}+\mathrm{v}^{\mathrm{p}} & (\mathrm{t}, \mathrm{x}) \in \mathrm{Q} \\
\mathrm{v}_{\mathrm{t}}=\Delta \mathrm{v}+\mathrm{w}^{\mathrm{q}} & (\mathrm{t}, \mathrm{x}) \in \mathrm{Q} \\
\mathrm{w}_{\mathrm{t}}=\Delta \mathrm{w}+\mathrm{u}^{\mathrm{r}} & (\mathrm{t}, \mathrm{x}) \in \mathrm{Q}
\end{array}\right.
$$

with $\mathrm{p}, \mathrm{q}, \mathrm{r}>0, \mathrm{n} \geq 0$ and nonnegative bounded continuous initial values. She proved that, if $\mathrm{pqr} \leq 1$, then any solution is global, while when pqr $>1$ and:

$$
\frac{\mathrm{n}}{2} \leq \frac{1}{\mathrm{pqr}-1} \max (1+\mathrm{p}+\mathrm{pq}, \quad 1+\mathrm{q}+\mathrm{rq}, \quad 1+\mathrm{r}+\mathrm{rp})
$$


then every nontrivial solution exhibits a finite blow-up time. She also uses Fujita's method. Rencławowicz (2000), she extended her study to a diagonal system of $n$ equations. The aim of this study is to establish new results on the blowing-up of solutions to systems of the following type Equation 1:

$$
\left\{\begin{array}{l}
\mathrm{u}_{\mathrm{t}}+|\mathrm{x}|^{\alpha}(-\Delta)^{\frac{\alpha_{1}}{2}}\left(\mathrm{a}_{11} \mathrm{u}\right)=\mathrm{h}(\mathrm{t}, \mathrm{x})|\mathrm{v}|^{\mathrm{p}} \\
\mathrm{v}_{\mathrm{t}}+|\mathrm{x}|^{\beta}\left\{(-\Delta)^{\frac{\alpha_{2}}{2}}\left(\mathrm{a}_{21} \mathrm{u}\right)+(-\Delta)^{\frac{\alpha_{3}}{2}}\left(\mathrm{a}_{22} \mathrm{v}\right)\right\}=\mathrm{k}(\mathrm{t}, \mathrm{x})|\mathrm{w}|^{\mathrm{q}} \\
\mathrm{w}_{\mathrm{t}}+|\mathrm{x}|^{\gamma}\left\{(-\Delta)^{\frac{\alpha_{4}}{2}}\left(\mathrm{a}_{31} \mathrm{u}\right)+(-\Delta)^{\frac{\alpha_{5}}{2}}\left(\mathrm{a}_{32} \mathrm{v}\right)+(-\Delta)^{\frac{\alpha_{6}}{2}}\left(\alpha_{33} \mathrm{w}\right)\right\} \\
=1(\mathrm{t}, \mathrm{x})|\mathrm{u}|^{\mathrm{r}} \mathrm{u}(0, \mathrm{x}) \geq 0, \quad \mathrm{v}(0, \mathrm{x}) \geq 0, \quad \mathrm{w}(0, \mathrm{x}) \geq 0
\end{array}\right.
$$

where, $\mathrm{p}, \mathrm{q}, \mathrm{r}>0$ and real, $\mathrm{a}_{\mathrm{ij}}$ are measurable, positive bounded functions and the nontrivial nonnegative functions h, $\mathrm{k}$ and 1 are assumed to satisfy Equation 2:

$$
\left\{\begin{array}{l}
0<\mathrm{h}\left(\mathrm{R}^{2} \tau, \mathrm{Ry}\right)=\mathrm{O}\left(\mathrm{R}^{\mu}\right) \\
0<\mathrm{k}\left(\mathrm{R}^{2} \tau, \mathrm{Ry}\right)=\mathrm{O}\left(\mathrm{R}^{\mathrm{k}}\right) \\
0<\mathrm{l}\left(\mathrm{R}^{2} \tau, \mathrm{Ry}\right)=\mathrm{O}\left(\mathrm{R}^{\lambda}\right)
\end{array}\right.
$$

The nonlocal operator $\Lambda^{\beta}=(-\Delta)^{\frac{\beta}{2}}$ is defined by:

$$
\Lambda^{\beta} \mathrm{v}(\mathrm{x})=\mathrm{F}^{-1}\left(|\xi|^{\beta} \mathrm{F}(\mathrm{v})(\xi)\right)(\mathrm{x})
$$

for every $v \in D\left(\Lambda^{\beta}\right)=H^{\beta}\left(\operatorname{IR}^{N}\right)$, where $H^{\beta}\left(I^{N}\right)$ is the homogeneous Sobolev space of order $\beta$ defined by:

$$
\begin{aligned}
& H^{\beta}\left(\operatorname{IR}^{N}\right)=\left\{u \in S^{\prime} ; \Lambda^{\beta} u \in L^{2}\left(I^{N}\right)\right\} \text { if } \beta \notin I N, \\
& H^{\beta}\left(\operatorname{IR}^{N}\right)=\left\{u \in L^{2}\left(\operatorname{IR}^{N}\right) ; \Lambda^{\beta} u \in L^{2}\left(I^{N}\right)\right\} \text { if } \beta \in I N
\end{aligned}
$$

where, $S^{\prime}$ is the space of Schwartz distributions; where $F$ denotes the Fourier transform and $\mathrm{F}^{-1}$ its inverse, $\mathrm{h}(\mathrm{x}, \mathrm{t})$ is positive function satisfies certain growth condition. The fractional Laplacian $\Lambda^{\beta}$ is related to Levy flights in physics. Many observations and experiments related to Levy flights (super-diffusion), e.g., collective slip diffusion on solid surfaces, quantum optics or Richardson turbulent diffusion, have been performed in recent years. The symmetric $\beta$-stable processes $(\beta \in(0$, 2)) are the basic characteristics for a class of jumping Levy's processes. Compared with the continuous Brownian motion $(\beta=2)$, symmetric $\beta$-stable processes have infinite jumps in an arbitrary time interval. The large jumps of these processes make their variances and expectations infinite according to $\beta \in(0,2)$ or $\beta \in(0,1]$, respectively. Let us also mention that when $\beta=\frac{3}{2}$, the symmetric $\beta$-stable processes appear in the study of stellar dynamics. The time fractional derivative has long been found to be very effective means to describe the anomalous attenuation behaviors. It is absolutely clear that our system (1) is more general than those cited above. We use a versatile method which has been employed by Pohozaev and Tesei (2000) and used by Hakem (2005). Before setting our theorem concerning (1), let us define the solutions we use.

\subsection{Main Results}

Let $\mathrm{L}_{\text {loc }}^{\mathrm{r}}\left((0, \mathrm{~T}) \times \mathrm{IR}^{\mathrm{n}}, 1 \mathrm{dtdx}\right)$ be the set of all functions $\mathrm{u}:(0, \mathrm{~T}) \times \mathrm{IR}^{\mathrm{n}} \rightarrow \operatorname{IR}$ such that $\int_{\mathrm{K}}|\mathrm{u}|^{\mathrm{r}} 1 \mathrm{dt} \mathrm{dx}<\infty$ for any compact $\mathrm{K} \subset(0, \mathrm{~T}) \times \mathrm{IR}^{\mathrm{n}}$.

\section{Definition 2.1:}

The 3-tuple (u, v, w) such that $\mathrm{u} \in \mathrm{C}([0, \mathrm{~T}]$; $\left.\mathrm{L}_{\text {loc }}^{1}\left(\operatorname{IR}^{\mathrm{n}}\right)\right) \cap \mathrm{C}\left([0, \mathrm{~T}] ; \mathrm{L}_{\text {loc }}^{1}\left((0, \mathrm{~T}) \times \mathrm{IR}^{\mathrm{n}}, 1 \mathrm{dtdx}\right)\right), \mathrm{v} \in \mathrm{C}([0$, $\left.\left.\mathrm{T}] ; \mathrm{L}_{\mathrm{loc}}^{1}\left(\mathrm{IR}^{\mathrm{n}}\right)\right) \cap \mathrm{C}\left([0, \mathrm{~T}] ; \mathrm{L}_{\mathrm{loc}}^{\mathrm{p}}((0, \mathrm{~T})) \mathrm{IR}^{\mathrm{n}}, \mathrm{h} \operatorname{dtdx}\right)\right)$ and $\mathrm{w} \in \mathrm{C}\left([0, \mathrm{~T}] ; \mathrm{L}_{\mathrm{loc}}^{1}\left(\operatorname{IR}^{\mathrm{n}}\right)\right) \cap \mathrm{C}\left([0, \mathrm{~T}] ; \mathrm{L}_{\mathrm{loc}}^{\mathrm{q}}((0, \mathrm{~T})) \mathrm{IR}^{\mathrm{n}}, \mathrm{k}\right.$ dtdx)) is called a solution to system (1) if:

$$
(\mathrm{H})\left\{\begin{array}{l}
\int_{\mathrm{Q}} \mathrm{h}|\mathrm{v}|^{\mathrm{p}} \zeta=-\int_{\mathrm{IR}^{\mathrm{n}}} \mathrm{u}_{0} \zeta(0)-\int_{\mathrm{Q}} \mathrm{u} \zeta_{\mathrm{t}}-\int_{\mathrm{Q}}|\mathrm{x}|^{\alpha} \mathrm{a}_{11} \mathrm{u}(-\Delta)^{\frac{\alpha_{1}}{2}} \zeta \\
\int_{\mathrm{Q}} \mathrm{k}|\mathrm{w}|^{\mathrm{q}} \zeta=-\int_{\mathbb{I R}^{\mathrm{n}}} \mathrm{v}_{0} \zeta(0)-\int_{\mathrm{Q}} \mathrm{v} \zeta_{\mathrm{t}}-\int_{\mathrm{Q}}|\mathrm{x}|^{\beta} \mathrm{a}_{21} \mathrm{u}(-\Delta)^{\frac{\alpha_{2}}{2}} \\
\zeta-\int_{\mathrm{Q}}|\mathrm{x}|^{\beta} \mathrm{a}_{22} \mathrm{v}(-\Delta)^{\frac{\alpha_{3}}{2}} \zeta \\
\left.\int_{\mathrm{Q}} \mathrm{l} \mathrm{u}\right|^{\mathrm{r}} \zeta=-\int_{\mathbb{I R}^{\mathrm{n}}} \mathrm{w}_{0} \zeta(0)-\int_{\mathrm{Q}} \mathrm{w} \zeta_{\mathrm{t}}-\int_{\mathrm{Q}}|\mathrm{x}|^{\gamma} \\
{\left[\begin{array}{l}
\left.\mathrm{a}_{31} \mathrm{u}(-\Delta)^{\frac{\alpha_{4}}{2}} \zeta+\mathrm{a}_{32} \mathrm{v}(-\Delta)^{\frac{\alpha_{5}}{2}} \zeta+\mathrm{a}_{33} \mathrm{w}(-\Delta)^{\frac{\alpha_{6}}{2}} \zeta\right]
\end{array}\right]}
\end{array}\right.
$$

for any nonnegative test function $\zeta \in \mathrm{C}_{0}^{2}\left(\mathrm{IR}^{+} \times \mathrm{IR}^{\mathrm{n}}\right)$ with $\zeta(\mathrm{T}, \mathrm{x})=0$. If $\mathrm{T}=+\infty$, we say that $(\mathrm{u}, \mathrm{v}, \mathrm{w})$ is a global weak solution.

Here, we require that the initial data are such that a local solution exists. Our result is.

\section{Theorem 2.1}

Let $(\mathrm{u}, \mathrm{v}, \mathrm{w})$ be a solution of (1) such that $\mathrm{u}_{0}, \mathrm{v}_{0}, \mathrm{w}_{0} \geq$ 0 . Let pqr $>1$ and: 


$$
\text { (K) } \mathrm{n} \leq \min \left\{\begin{array}{l}
\frac{\mathrm{r}\left[\mu+\alpha_{1}-\alpha+\mathrm{p}\left(\alpha_{3}-\beta-2 \mathrm{q}+\kappa+\mathrm{q} \alpha_{6}-\mathrm{q} \gamma\right)\right]+\lambda+2}{\mathrm{pqr}-1}, \frac{\mathrm{r}\left(\alpha_{4}-\gamma-2\right)+\lambda+2}{\mathrm{r}-1}, \\
\frac{\mathrm{r}\left[\alpha_{2}+\kappa-\beta+\mathrm{q}\left(\alpha_{6}-\gamma-2\right)\right]+\lambda+2}{\mathrm{qr}-1}, \frac{\mathrm{r}\left[\mu+\alpha_{1}-\alpha+\mathrm{p}\left(\alpha_{5}-\gamma-2\right)\right]+\lambda+2}{\mathrm{pr}-1}
\end{array}\right\}
$$

Then every nontrivial solution of (1) blows up in finite time.

\section{Proof}

The proof is by contradiction. Let $(\mathrm{u}, \mathrm{v}, \mathrm{w})$ be a global solution of (1) with pqr > 1 and suppose (K) is satisfied. Let $\zeta$ be a nonnegative test function such that:

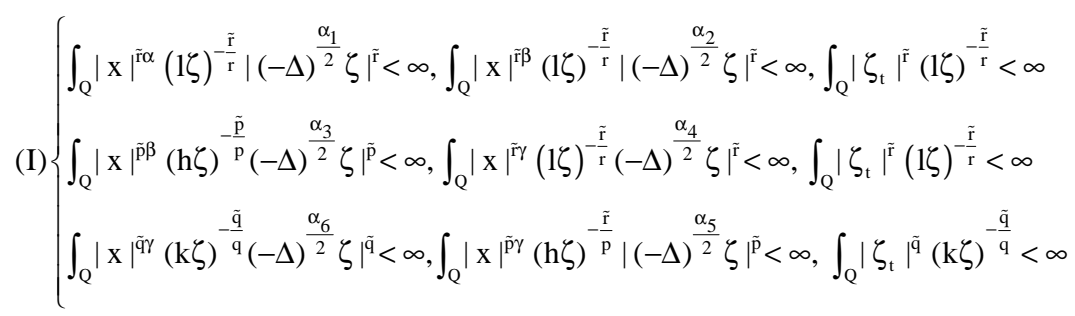

As $u(0, x), v(0, x), w(0, x) \geq 0$, using $(H)$ we have, for $\zeta \geq 0$ Equation 3-5:

$$
\begin{aligned}
& \int_{Q} \mathrm{~h}|\mathrm{v}|^{p} \zeta \leq \int_{Q}\left|\mathrm { u } \left\|\left.\zeta_{\mathrm{t}}\left|+\left\|\alpha_{11}\right\|_{\infty} \int_{Q}\right| \mathrm{x}\right|^{\alpha}\left|\mathrm{u} \|(-\Delta)^{\frac{\alpha_{1}}{2}} \zeta\right|\right.\right. \\
& \int_{Q} \mathrm{k}|\mathrm{w}|^{q} \zeta \leq \int_{Q}\left|\mathrm{v}\left\|\left.\zeta_{\mathrm{t}}\left|+\left\|\alpha_{21}\right\|_{\infty} \int_{Q}\right| \mathrm{x}\right|^{\beta} \mid \mathrm{u}\right\|(-\Delta)^{\frac{\alpha_{2}}{2}}\right. \\
& \left.\zeta\left|+\left\|\mathrm{a}_{22}\right\|_{\infty} \int_{Q}\right| \mathrm{x}\right|^{\gamma}\left|\mathrm{v} \|(-\Delta)^{\frac{\alpha_{3}}{2}} \zeta\right| \\
& \int_{Q} 1|\mathrm{u}|^{r} \zeta \leq \int_{Q}\left|\mathrm { w } \left\|\zeta _ { \mathrm { t } } | + \| \mathrm { a } _ { 3 1 } \| _ { \infty } \int _ { Q } | \mathrm { x } | ^ { \gamma } \left|\mathrm{u}\left\|(-\Delta)^{\frac{a_{4}}{2}} \zeta \mid+\right\| \mathrm{a}_{32} \|_{\infty}\right.\right.\right. \\
& \int_{Q}|\mathrm{x}|^{\gamma}\left|\mathrm { v } \left\|\left.(-\Delta)^{\frac{\mathrm{a}_{5}}{2}} \zeta\left|+\left\|\mathrm{a}_{33}\right\|_{\infty} \int_{Q}\right| \mathrm{x}\right|^{\gamma}\left|\mathrm{w} \|(-\Delta)^{\frac{\alpha_{6}}{2}} \zeta\right|\right.\right.
\end{aligned}
$$

where, $\quad\left\|\mathrm{a}_{21}\right\|_{\infty}=\max _{\mathrm{t}, \mathrm{x}} \| \mathrm{a}_{21} \mid$. To estimate $\int_{\mathrm{Q}}\left|\mathrm{u} \| \zeta_{\mathrm{t}}\right|$, we observe that it can be written as:

$$
\int_{Q}|u|\left|\zeta_{\mathrm{t}}\right|=\int_{Q}|\mathrm{u}|(1 \zeta)^{\frac{1}{r}}\left|\zeta_{\mathrm{t}}\right|(1 \zeta)^{-\frac{1}{r}}
$$

using Holder inequality, we have with $\frac{1}{\tilde{\mathrm{r}}}+\frac{1}{\mathrm{r}}=1$ Equation 6 and 7:

$$
\begin{aligned}
& \int_{Q}\left|\mathrm{u} \| \zeta_{\mathrm{t}}\right| \leq\left(\int_{Q} 1|\mathrm{u}|^{\mathrm{r}} \zeta\right)^{\frac{1}{r}}\left(\int_{Q}\left|\zeta_{\mathrm{t}} \tilde{\mid}\right|^{\tilde{r}}(1 \zeta)^{\frac{\tilde{r}}{\mathrm{r}}}\right)^{\frac{1}{\tilde{r}}} \\
& \int_{Q}|\mathrm{x}|^{\alpha}\left|\mathrm{u} \|(-\Delta)^{\frac{\alpha_{1}}{2}} \zeta\right| \leq\left(\int_{Q} 1|\mathrm{u}|^{\mathrm{r}} \zeta\right)^{\frac{1}{r}}\left(\int_{Q}|\mathrm{x}|^{\tilde{\alpha} \alpha}(1 \zeta)^{-\frac{\tilde{r}}{r}}\left|(-\Delta)^{\frac{\alpha_{1}}{2}} \zeta\right|^{\tilde{r}}\right)^{\frac{1}{\tilde{r}}}
\end{aligned}
$$

Next, we set:

$$
\begin{aligned}
& A_{\alpha, \alpha 1}^{\mathrm{r}, 1}=\left(\int_{Q}\left|\zeta_{\mathrm{t}}\right|^{\tilde{r}}(1 \zeta)^{-\frac{\tilde{r}}{\mathrm{r}}}\right)^{\frac{1}{\tilde{r}}}+C\left(\int_{Q}|x|^{\tilde{\alpha} \alpha}(1 \zeta)^{-\frac{\tilde{r}}{\mathrm{r}}}\left|(-\Delta)^{\frac{\alpha_{1}}{2}} \zeta\right|^{\tilde{f}}\right)^{\frac{1}{\tilde{r}}} \\
& =\mathrm{A}_{\mathrm{r}, \mathrm{l}}^{(1)}+\mathrm{CA}_{\alpha, \alpha_{1}, \mathrm{r}, 1}^{(2)}
\end{aligned}
$$

And:

$$
X=\left(\left.\int_{Q}|| u\right|^{r} \zeta\right)^{\frac{1}{r}} \text { and } Y=\left(\int_{Q} h|v|^{p} \zeta\right)^{\frac{1}{p}}
$$

Then using (6) and (7) in (3), we obtain Equation 8:

$$
\mathrm{Y}^{\mathrm{p}} \leq \mathrm{XA}_{\alpha, \alpha_{1}}^{\mathrm{r}, 1}
$$

We also have:

$$
\int_{Q} \mathrm{k}|\mathrm{w}|^{q} \zeta \leq\left(\int_{Q} \mathrm{~h}|\mathrm{v}|^{\mathrm{p}} \zeta\right)^{\frac{1}{\mathrm{p}}} \mathrm{A}_{\beta, \alpha_{3}}^{\mathrm{p}, \mathrm{h}}+\mathrm{C}\left(\int_{\mathrm{Q}} 1|\mathrm{u}|^{\mathrm{r}} \zeta\right)^{\frac{1}{\mathrm{r}}} \mathrm{A}_{\beta, \alpha_{2}, \mathrm{r}, 1}^{(2)}
$$

And:

$$
\begin{aligned}
& \int_{Q} 1|\mathrm{u}|^{\mathrm{r}} \zeta \leq\left(\int_{Q} \mathrm{k}|\mathrm{w}|^{q} \zeta\right)^{\frac{1}{q}} \mathrm{~A}_{\gamma, \alpha_{6}}^{\mathrm{q}, \mathrm{k}}+\mathrm{C}\left(\int_{Q} 1|\mathrm{u}|^{\zeta} \zeta\right)^{\frac{1}{r}} \\
& \mathrm{~A}_{\gamma, \alpha_{4}, \mathrm{r}, 1}^{(2)}+\mathrm{C}\left(\int_{Q} \mathrm{~h}|\mathrm{v}|^{p} \zeta\right)^{\frac{1}{\mathrm{p}}} \mathrm{A}_{\gamma, \alpha_{5}, \mathrm{p}, \mathrm{h}}^{(2)}
\end{aligned}
$$

If we $\operatorname{set} Z=\left(\int_{Q} k|w|^{q} \zeta\right)^{\frac{1}{q}}$ then we can write Equation 9 and 10: 


$$
\begin{aligned}
& \mathrm{Z}^{\mathrm{q}} \leq \mathrm{Y} \mathrm{A}_{\beta, \alpha_{3}}^{\mathrm{p}, \mathrm{h}}+\mathrm{CX} \mathrm{A}_{\beta, \alpha_{2}, \mathrm{r}, 1}^{(2)} \\
& \mathrm{X}^{\mathrm{r}} \leq \mathrm{ZA}_{\gamma, \alpha_{6}}^{\mathrm{q}, \mathrm{k}}+\mathrm{CX} \mathrm{A}_{\gamma, \alpha_{4}, \mathrm{r}, 1}^{(2)}+\mathrm{CY} \mathrm{A}_{\gamma, \alpha_{5}, \mathrm{p}, \mathrm{h}}^{(2)}
\end{aligned}
$$

So Equation 11 and 12:

$$
\begin{aligned}
& \mathrm{Z}^{\mathrm{pq}} \leq \mathrm{C} \mathrm{Y}^{\mathrm{p}}\left[\mathrm{A}_{\beta, \alpha_{3}}^{\mathrm{p}, \mathrm{h}}\right]^{\mathrm{p}}+\mathrm{C} \mathrm{X}^{\mathrm{p}}\left[\mathrm{A}_{\beta, \alpha_{2}, \mathrm{r}, 1}^{(2)}\right]^{\mathrm{p}} \\
& \mathrm{X}^{\mathrm{rqq}} \geq \mathrm{C} \mathrm{Z}^{\mathrm{pq}}\left[\mathrm{A}_{\gamma, \alpha_{6}}^{\mathrm{q}, \mathrm{k}}\right]^{\mathrm{pq}}+\mathrm{C} \mathrm{X}^{\mathrm{pq}}\left[\mathrm{A}_{\gamma, \alpha_{4} \mathrm{r}, 1}^{(2)}\right]^{\mathrm{pq}} \\
& +\mathrm{C}^{\mathrm{pq}}\left[\mathrm{A}_{\gamma, \alpha_{5}, \mathrm{p}, \mathrm{h}}^{(2)}\right]^{\mathrm{pq}}
\end{aligned}
$$

Inserting (11) in (12), we get Equation 13:

$$
\begin{aligned}
& \mathrm{X}^{\mathrm{rpq}} \leq \mathrm{C} \mathrm{Y}^{\mathrm{p}}\left[\mathrm{A}_{\gamma, \alpha_{6}}^{\mathrm{q}, \mathrm{k}}\right]^{\mathrm{pq}}\left[\mathrm{A}_{\beta, \alpha_{3}}^{\mathrm{p}, \mathrm{h}}\right]^{\mathrm{p}} \\
& +\mathrm{CX}^{\mathrm{p}}\left[\mathrm{A}_{\gamma, \alpha_{6}}^{\mathrm{q}, \mathrm{k}}\right]^{\mathrm{pq}}\left[\mathrm{A}_{\beta, \alpha_{2}, \mathrm{r}, 1}^{(2)}\right]^{\mathrm{p}} \\
& +\mathrm{CX}^{\mathrm{pq}}\left[\mathrm{A}_{\gamma, \alpha_{4}, \mathrm{r}, \mathrm{l}}^{(2)}\right]^{\mathrm{pq}}+\mathrm{C} \mathrm{Y}^{\mathrm{pq}}\left[\mathrm{A}_{\gamma, \alpha_{5}, \mathrm{p}, \mathrm{h}}^{(2)}\right]^{\mathrm{pq}}
\end{aligned}
$$

Using (8) in (13), we obtain:

$$
\begin{aligned}
& \mathrm{X}^{\mathrm{rpq}} \leq \mathrm{CX}\left[\mathrm{A}_{\alpha, \alpha_{1}}^{\mathrm{r}, \mathrm{l}}\right]\left[\mathrm{A}_{\gamma, \alpha_{6}}^{\mathrm{q}, \mathrm{k}}\right]^{\mathrm{pq}}\left[\mathrm{A}_{\beta, \alpha_{3}}^{\mathrm{p}, \mathrm{h}}\right]^{\mathrm{p}} \\
& +\mathrm{CXX}^{\mathrm{p}}\left[\mathrm{A}_{\gamma, \alpha_{6}}^{\mathrm{q}, \mathrm{k}}\right]^{\mathrm{pq}}\left[\mathrm{A}_{\beta, \alpha_{2}, \mathrm{r}, 1}^{(2)}\right]^{\mathrm{p}} \\
& +\mathrm{CXX}^{\mathrm{pq}}\left[\mathrm{A}_{\gamma, \alpha_{4}, \mathrm{r}, 1}^{(2)}\right]^{\mathrm{pq}}+\mathrm{CX}^{\mathrm{q}}\left[\mathrm{A}_{\gamma, \alpha_{5}, \mathrm{p}, \mathrm{h}}^{(2)}\right]^{\mathrm{pq}}\left[\mathrm{A}_{\alpha, \alpha_{1}}^{\mathrm{r}, 1}\right]^{\mathrm{q}}
\end{aligned}
$$

which we write as Equation 14:

$$
\mathrm{X}^{\mathrm{pqr}-1} \leq \mathrm{a}+\mathrm{bX}^{\mathrm{p}-1}+\mathrm{cX}^{\mathrm{pq}-1}+\mathrm{dX} \mathrm{q}^{\mathrm{q}-1}
$$

With:

$$
\begin{aligned}
& \mathrm{a}=\mathrm{C}\left[\mathrm{A}_{\alpha, \alpha_{1}}^{\mathrm{r}, \mathrm{l}}\right]\left[\mathrm{A}_{\gamma, \alpha_{6}}^{\mathrm{q}, \mathrm{k}}\right]^{\mathrm{pq}}\left[\mathrm{A}_{\beta, \alpha_{3}}^{\mathrm{p}, \mathrm{h}}\right]^{\mathrm{p}}, \mathrm{b}=\mathrm{C}\left[\mathrm{A}_{\gamma, \alpha_{6}}^{\mathrm{q}, \mathrm{k}}\right]^{\mathrm{pq}}\left[\mathrm{A}_{\beta, \alpha_{2}, \mathrm{r}, 1}^{(2)}\right]^{\mathrm{p}} \\
& \mathrm{c}=\mathrm{C}\left[\mathrm{A}_{\gamma, \alpha_{4}, \mathrm{r}, \mathrm{l}}^{(2)}\right]^{\mathrm{pq}}, \quad \mathrm{d}=\mathrm{C}\left[\mathrm{A}_{\gamma, \alpha_{5}, \mathrm{p}, \mathrm{h}}^{(2)}\right]^{\mathrm{pq}}\left[\mathrm{A}_{\alpha, \alpha_{1}}^{\mathrm{r}, \mathrm{l}}\right]^{\mathrm{q}}
\end{aligned}
$$

Here $\mathrm{C}$ denotes a constant that may change in different occurrences. Now, by using the $\varepsilon$-Young inequality, we obtain Equation 15:

$$
\mathrm{b} \mathrm{X}^{\mathrm{p}-1} \leq \varepsilon \mathrm{X}^{\mathrm{pqr}-1}+\mathrm{C}(\varepsilon) \mathrm{b}^{\mathrm{m}_{1}} ; \mathrm{m}_{1}=\frac{\mathrm{pqr}-1}{\mathrm{p}-1}, \frac{1}{\mathrm{~m}_{1}}+\frac{1}{\mathrm{~m}_{1}}=1
$$

Similarly Equation 16 and 17:

$$
\begin{aligned}
& \mathrm{cX}^{\mathrm{pq}-1} \leq \varepsilon \mathrm{X}^{\mathrm{pqr}-1}+\mathrm{C}(\varepsilon) \mathrm{b}^{\mathrm{m}_{2}} ; \mathrm{m}_{2}=\frac{\mathrm{pqr}-1}{\mathrm{pq}-1}, \frac{1}{\mathrm{~m}_{2}}+\frac{1}{\mathrm{~m}_{2}}=1 \\
& \mathrm{dX}^{\mathrm{q}-1} \leq \varepsilon \mathrm{X}^{\mathrm{pqr}-1}+\mathrm{C}(\varepsilon) \mathrm{d}^{\mathrm{m}_{3}} ; \mathrm{m}_{3}=\frac{\mathrm{pqr}-1}{\mathrm{p}-1}, \frac{1}{\mathrm{~m}_{3}}+\frac{1}{\mathrm{~m}_{3}}=1
\end{aligned}
$$

$\mathrm{C}(\varepsilon)$ has a different meaning in 15,16 and 17. Taking $\varepsilon$ small enough and using 15-17 in 14, we obtain Equation 18:

$$
(1-3 \varepsilon) X^{\mathrm{pqr}-1} \leq \mathrm{a}+\mathrm{C}(\varepsilon)\left[\mathrm{b}^{\mathrm{m}_{1}}+\mathrm{c}^{\mathrm{m}_{2}}+\mathrm{d}^{\mathrm{m}_{3}}\right]
$$

Next, we consider $\phi \in \mathrm{C}^{2}\left(\mathrm{IR} ; \mathrm{IR}^{+}\right)$such that:

$$
\phi(r)=\left\{\begin{array}{lll}
1 & \text { for } & r \leq 1 \\
0 & \text { for } & r \geq 2
\end{array}\right.
$$

and $0 \leq \phi \leq 1$ for any $r>0$. If we set:

$$
\zeta(t, x)=\phi^{\theta}\left(\frac{t+|x|^{2}}{R^{2}}\right), \quad R>0
$$

and take $\theta$ large enough, we ensure the validity of the requirement (I) at the beginning of the proof. At this stage, we introduce the scaled variables:

$$
\tau=\mathrm{tR}^{-2}, \quad \mathrm{y}=\mathrm{x} \mathrm{R}^{-1}
$$

We have:

$$
\mathrm{a} \leq \mathrm{CR}^{\mathrm{s}_{\mathrm{a}}}, \quad \mathrm{b} \leq \mathrm{CR}^{\mathrm{s}_{\mathrm{b}},}, \mathrm{c} \leq \mathrm{C} \mathrm{R}^{\mathrm{s}_{\mathrm{c}}} \quad \mathrm{d} \leq \mathrm{CR}^{\mathrm{s}_{\mathrm{d}}}
$$

Where:

$$
\begin{aligned}
& \mathrm{s}_{\mathrm{a}}=\left[\alpha-\alpha_{1}-\frac{\lambda}{\mathrm{r}}+\frac{(2+\mathrm{n})(\mathrm{r}+1)}{\mathrm{r}}\right] \\
& +\mathrm{pq}\left[\gamma-\alpha_{6}-\frac{\mathrm{k}}{\mathrm{q}}+\frac{(2+\mathrm{n})(\mathrm{q}+1)}{\mathrm{q}}\right] \\
& +\mathrm{p}\left[\beta-\alpha_{3}-\frac{\mathrm{u}}{\mathrm{p}}+\frac{(2+\mathrm{n})(\mathrm{q}+1)}{\mathrm{p}}\right] \\
& \mathrm{s}_{\mathrm{b}}=\mathrm{p}\left[\mathrm{q} \gamma-\mathrm{q} \alpha_{6}-\kappa+(2+\mathrm{n})(\mathrm{q}+1)\right] \\
& +\mathrm{p}\left[\beta-\alpha_{2}-\frac{\lambda}{\mathrm{r}}+\frac{(2+\mathrm{n})(\mathrm{r}-1)}{\mathrm{r}}\right]
\end{aligned}
$$




$$
\begin{aligned}
& \mathrm{s}_{\mathrm{c}}=\mathrm{pq}\left[\gamma \mid-\frac{\lambda}{\mathrm{r}}-\alpha_{4}+\frac{(2+\mathrm{n})(\mathrm{r}+1)}{\mathrm{r}}\right] \\
& \mathrm{s}_{\mathrm{d}}=\mathrm{q}\left[\mathrm{p} \gamma-\mathrm{p} \alpha_{5}-\mu+(2+\mathrm{n})(\mathrm{p}+1)\right] \\
& +\mathrm{q}\left[\alpha-\alpha_{1}-\frac{\lambda}{\mathrm{r}}+\frac{(2+\mathrm{n})(\mathrm{r}-1)}{\mathrm{r}}\right]
\end{aligned}
$$

Now, we require:

$$
\mathrm{s}_{\mathrm{a}} \leq 0, \quad \mathrm{~s}_{\mathrm{b}} \leq 0, \quad \mathrm{~s}_{\mathrm{c}} \leq 0, \quad \mathrm{~s}_{\mathrm{d}} \leq 0
$$

which are, respectively, equivalent to:
$\mathrm{n} \leq \frac{\lambda+2+\mathrm{r}\left[\mu+\alpha_{1}-\alpha+\mathrm{p}\left(\alpha_{3}-\beta-2 \mathrm{q}+\kappa+\mathrm{q} \alpha_{6}-\mathrm{q} \gamma\right)\right]}{\mathrm{pqr}-1}$
$\mathrm{n} \leq \frac{\lambda+2+\mathrm{r}\left[\alpha_{2}+\kappa-\beta+\mathrm{q}\left(\alpha_{6}-\gamma-2\right)\right]}{\mathrm{qr}-1}$
$\mathrm{n} \leq \frac{\lambda+2+\mathrm{r}\left(\alpha_{4}-\gamma-2\right)}{\mathrm{r}-1}$
$\mathrm{n} \leq \frac{\lambda+2+\mathrm{r}\left[\mu+\alpha_{1}-\alpha+\mathrm{p}\left(\alpha_{5}-\gamma-2\right)\right]}{\mathrm{pr}-1}$

in other words:

$$
\begin{aligned}
& \mathrm{n} \leq \min \left\{\frac{\mathrm{r}\left[\mu+\alpha_{1}-\alpha+\mathrm{p}\left(\alpha_{3}-\beta-2 \mathrm{q}+\kappa+\mathrm{q} \alpha_{6}-\mathrm{q} \gamma\right)\right]+\lambda+2}{\mathrm{pqr}-1}, \frac{\mathrm{r}\left(\alpha_{4}-\gamma-2\right)+\lambda+2}{\mathrm{r}-1},\right. \\
& \left.\frac{\mathrm{r}\left[\alpha_{2}+\kappa-\beta+\mathrm{q}\left(\alpha_{6}-\gamma-2\right)\right]+\lambda+2}{\mathrm{qr}-1}, \frac{\mathrm{r}\left[\mu+\alpha_{1}-\alpha+\mathrm{p}\left(\alpha_{5}-\gamma-2\right)\right]+\lambda+2}{\mathrm{pr}-1}\right\}
\end{aligned}
$$

We have two cases:

- Either $\mathrm{s}_{\mathrm{a}}<0, \mathrm{~s}_{\mathrm{b}}<0, \mathrm{~s}_{\mathrm{c}}<0$ and $\mathrm{s}_{\mathrm{d}}<0$. In this case, we let $\mathrm{R} \rightarrow \infty$ in (18) to obtain:

$$
\lim _{\mathrm{R} \rightarrow+\infty} \mathrm{X}^{\mathrm{pqr}-1}=0
$$

Hence $\mathrm{u} \equiv 0$; this implies via (8) that $\mathrm{v} \equiv 0$ and finally $\mathrm{w} \equiv 0$ from (9). That is a contradiction

- Or $\mathrm{s}_{\mathrm{a}}<0$ or $\mathrm{s}_{\mathrm{b}}<0$ or $\mathrm{s}_{\mathrm{c}}<0$ or $\mathrm{s}_{\mathrm{d}}<0$, i.e., at least one of the exponents is not zero. In this case, we get:

$$
\lim _{\mathrm{R} \rightarrow+\infty} \mathrm{X}^{\mathrm{pqr}-1} \leq \mathrm{C}<\infty
$$

So:

$\lim _{\mathrm{R} \rightarrow+\infty} \int_{\Omega_{\mathrm{R}}} 1|\mathrm{u}|^{\mathrm{r}} \zeta=0, \quad$ where $\Omega_{\mathrm{R}}=\left\{(\mathrm{t}, \mathrm{x}): \mathrm{R}^{2} \leq \mathrm{t}+|\mathrm{x}|^{2} \leq 2 \mathrm{R}^{2}\right\}$

Now we write (8) in the form:

$$
\begin{aligned}
\int_{\Omega_{\mathrm{R}}} \mathrm{h}|\mathrm{v}|^{\mathrm{r}} \zeta \leq\left(\int_{\Omega_{\mathrm{R}}} 1|\mathrm{u}|^{\mathrm{r}} \zeta\right) \mathrm{A}_{\alpha, \alpha_{1}}^{\mathrm{r}, \mathrm{l}} \quad \text { where: } \\
\sigma_{1}=\left[\alpha-\alpha_{1}-\frac{\lambda}{\mathrm{r}}+\frac{(2+\mathrm{n})(\mathrm{r}-1)}{\mathrm{r}}\right]+\mathrm{p}\left[\mathrm{q} \gamma-\kappa-\mathrm{q} \alpha_{6}+(2+\mathrm{n})(\mathrm{q}-1)\right]+\left[\mathrm{p} \beta-\mu-\mathrm{p} \alpha_{3}+(2+\mathrm{n})(\mathrm{p}-1)\right] \\
\sigma_{2}=\left[\beta-\alpha_{3}-\frac{\mathrm{u}}{\mathrm{p}}+\frac{(2+\mathrm{n})(\mathrm{p}-1)}{\mathrm{p}}\right]+\mathrm{q}\left[\mathrm{r} \alpha-\lambda-\mathrm{r} \alpha_{1}+(2+\mathrm{n})(\mathrm{r}-1)\right]+\left[\mathrm{q} \gamma-\kappa-\mathrm{q} \alpha_{6}+(2+\mathrm{n})(\mathrm{q}-1)\right] \\
\sigma_{3}=\left[\gamma-\alpha_{6}-\frac{\mathrm{k}}{\mathrm{q}}+\frac{(2+\mathrm{n})(\mathrm{q}-1)}{\mathrm{q}}\right]+\mathrm{r}\left[\mathrm{p} \beta-\mu-\mathrm{p} \alpha_{3}+(2+\mathrm{n})(\mathrm{p}-1)\right]+\left[\mathrm{r} \alpha-\lambda-\mathrm{r} \alpha_{1}+(2+\mathrm{n})(\mathrm{r}-1)\right]
\end{aligned}
$$


The choice of $\sigma_{1} \leq 0, \sigma_{2} \leq 0$ and $\sigma_{3} \leq 0$ leads to:

$\mathrm{n} \leq \frac{(\lambda+2)+\mathrm{r}\left[\mu+\alpha_{1}-\alpha+\mathrm{p} \alpha_{3}-\mathrm{p} \beta-2 \mathrm{pq}+\mathrm{pq} \alpha_{6}+\mathrm{p \kappa}-\mathrm{pq} \gamma\right]}{\mathrm{pqr}-1}$

$\mathrm{n} \leq \frac{(\mu+2)+\mathrm{p}\left[\alpha_{3}+\kappa-\beta+\mathrm{q}\left(\alpha_{6}-\gamma+\lambda\right)+\mathrm{rq}\left(\alpha_{1}-\alpha-2\right)\right]}{\mathrm{pqr}-1}$

$\mathrm{n} \leq \frac{(\kappa+2)+\mathrm{q}\left[\lambda+\alpha_{6}-\gamma+\mathrm{r}\left(\alpha_{1}-\alpha+\mu-\mathrm{p} \beta+\mathrm{p} \alpha_{3}-2 \mathrm{p}\right)\right]}{\mathrm{pqr}-1}$

Now, if we take the case studied by (Igbida and Kirane, 2002): $\mathrm{a}=\beta=\gamma=0$ and $=\mathrm{a}_{1}=\mathrm{a}_{3}=\mathrm{a}_{6}=2$, we obtain the same results as they did:

$$
\mathrm{n} \leq \min \left\{\frac{\lambda+2+\mathrm{r}[\mu+2+\mathrm{p}(\kappa+2)]}{\mathrm{pqr}-1}, \frac{\mu+2+\mathrm{p}[\mathrm{q}(\lambda+2)+(\kappa+2)]}{\mathrm{pqr}-1},\right.
$$

If we take the case studied by (Renclawowicz, 1998): $\mathrm{a}=\beta=\gamma=\lambda=\mu=\mathrm{k}=0$ and $\mathrm{a}_{1}=\mathrm{a}_{3}=\mathrm{a}_{6}=2$, we obtain the same results as she did:

$$
\mathrm{n} \leq \frac{2}{\mathrm{pqr}-1} \min \{\mathrm{pq}+\mathrm{p}+1, \mathrm{rp}+\mathrm{r}+1, \mathrm{qr}+\mathrm{q}+1\}
$$

\section{REFERENCES}

Bandle, C. and H. Brunner, 1998. Blow-up in diffusion equations: A survey. J. Comput. Applied Math., 97: 3-22. DOI: 10.1016/S0377-0427(98)00100-9

Escobedo, M. and M.A. Herrero, 1991. Boundedness and blow up for a semilinear reaction-diffusion system. J. Differential Eq., 89: 176-202. DOI: 10.1016/00220396(91)90118-S
Fila, M., H.A. Levine and Y. Uda, 1994. A Fujita-type global existence-global non-existence theorem for a system of reaction diffusion equations with differing diffusivities. Math. Meth. Applied Sci., 17: 807-835. DOI: $10.1002 / \mathrm{mma} .1670171005$

Fujita, H., 1966. On the blowing up of solutions of the Cauchy problem for $\mathrm{u}_{\mathrm{t}}=\Delta \mathrm{u}+\mathrm{u}^{1+\alpha}$. J. Fac. Sci. Univ. Tokyo, Sect., I13: 109-124.

Hakem, A., 2005. Blow-up results for evolution problems on $\mathrm{IR}^{\mathrm{n}}$. Math. Nachr., 278: 1033-1040.

Igbida, N. and M. Kirane, 2002. Blow up for a completely coupled Fujita type reaction-diffusion system. Colloq. Math., 92: 87-96. DOI: $10.4064 / \mathrm{cm} 92-1-8$

Levine, H.A., 1990. The role of critical exponents in blowup theorems. SIAM Rev., 32: 262-288. DOI: $10.1137 / 1032046$

OSEU, 1968. On some existence and uniqueness theorems for nonlinear parabolic equations. Proc. Sympos. Pure Math. 18, Part I, Amer. Math. Soc., 138-161.

Pohozaev, S.I. and A. Tesei, 2000. Blow-up of nonegative solutions to quasilinear parabolic Inequalities. Math. Appl., 11: 99-109.

Renclawowicz, J., 1998. Global existence and blow up of solutions for a completely coupled Fujita type system of reaction-diffusion equations. Applied Math., 25: 313-326.

Rencławowicz, J., 2000. Global existence and blow-up for a completely coupled Fujita type system. Applic. Math., 27: 203-218.

Samarskii, A.A., V.A. Galaktinov, S.P. Kurdyumov and A.P. Mikhailov, 1995. Blow-Up in Quasilinear Parabolic Equations. 1st Edn., Walter de Gruyter, Berlin, ISBN-10: 3110889862, pp: 533. 